\title{
Material plastic properties characterization by coupling experimental and numerical analysis of small punch beam tests
}

\author{
Xingguo Zhou, Wenke Pan *, Donald Mackenzie \\ Department of Mechanical Engineering, University of Strathclyde, Glasgow, UK
}

\section{A R T I C L E I N F O}

\section{Article history:}

Received 25 June 2012

Accepted 2 July 2012

Available online 31 July 2012

\section{Keywords:}

Small punch beam test

Plastic properties characterization

Genetic algorithm

Finite element analysis

\begin{abstract}
A B S T R A C T
A novel small punch beam testing (SPBT) system consisting of a top die, a bottom die and a flat punch with semi-circular cross-section head has been designed and tested. The specimen is a small beam with rectangular cross-section. This SPBT method has the advantage over conventional tensile testing for much less material required for testing and also over the traditional small punch testing because not only less material required but also high accuracy to make the punch head. Through coupling the numerical modelling and experimental results, genetic algorithm has been employed to successfully characterize the material plastic properties.
\end{abstract}

(c) 2012 Elsevier Ltd. All rights reserved.

\section{Introduction}

The small punch testing (SPT) technique was developed to characterize material properties in the late 1970s [1]. In the test, a cylindrical punch with a hemispherical head deforms a thin disc shape specimen, which is clamped between two co-axial hollow cylindrical dies. The thickness of the sample can be as small as $0.5 \mathrm{~mm}$ or $0.25 \mathrm{~mm}$ and the diameter is about 6 to $10 \mathrm{~mm}$. Comparison of SPT results with conventional tensile testing (CTT) method is very encouraging. To date, material properties that have been evaluated by SPT include elastic modulus, plastic deformation properties such as yield stress and tensile strength [1-5], creep properties [6-8] and fracture properties [9-11], etc.

Several national associations have considered or are currently investigating testing standards for SPT. The Japan Atomic Energy Research Institute has produced practice recommendations for small punch (SP) testing of metallic Materials in 1988 [12]. CEN published Small Punch Test Method for Metallic Materials Part A: A Code of Practice for Small Punch Creep in 2005 [13] and Part B: A Code of Practice for Small Punch Testing for Tensile and Fracture Behavior in 2006 [14]. The American Society for Testing and Materials has issued a standard test method for small punch testing of ultra-high molecular weight polyethene used in surgical implants in 2008 [15]. The progress [16] of standardization of small punch test in China was reported in 2010.

One practical advantage of SPT over conventional tensile testing is that the specimen size is relatively small and therefore only

\footnotetext{
* Corresponding author.

E-mail address: wenke.pan@strath.ac.uk (W. Pan).
}

small quantity of material is required for testing. This is particularly useful when testing material from operating plants for life extension programs, as the small amount of material required can often be removed from the surface of components without plant shutdown and intrusive welded repairs are not required afterwards. One disadvantage of SPT is the difficulty and expense of manufacturing the $2.5 \mathrm{~mm}$ diameter hemi-spherical head punch to the required dimensional accuracy. To overcome this problem, an alternative Small Punch Plane Strain (SPPS) test system with a cylindrical punch contact surface and rectangular test specimen was proposed [17]. This has been successfully used to characterize material's plastic properties, with Ramberg-Osgood constitutive relationship employed. Recently, instead of using rectangular specimens, Sehgal et. al [18] used miniature samples for their punch tests. The yield strength and fracture toughness of die steel D3 and Chromium steel H11 were evaluated and compared with the values obtained from standard tests. Their simulated load-displacement curves were in good agreement with the results obtained experimentally, however, as the distance between the lower dies is just a little bit larger than the thickness of the specimen the deformation in the specimen is quite complex.

To characterize material properties using the small punch testing method, trial and error methods, a genetic algorithm method [17] and neural network method [19], etc. have all been used. In this paper, a small punch testing system based on a small beam shape specimen and punch similar to that of SPPS is presented. Material properties are evaluated from the deformation through comparison with multi-linear strain hardening Finite Element analysis of specimen deformation and a genetic algorithm utilizing a cost function based on the relative difference between the 
experimental and testing forces at the top centre of the beam. The small punch beam testing tool design will be presented in the Section 2, while experiment procedure will be given in Section 3. FEM modelling is presented in Section 4, material property characterization using a genetic algorithm method in Section 5 and results in Section 6.

\section{Small punch beam testing tool design}

The standard small punch test (SPT) tool employs an upper and a lower hollow cylindrical shape die and a cylindrical punch with hemispherical punch head. To avoid the high cost and low accuracy for manufacturing hemispherical punch head, the new designed tool testing system shown in Fig. 1 has been developed. The tool consists of top and bottom blocks, each having a narrow through slot. The test beam specimen is located in a shallow slot perpendicular to the through slot. The punch is a rectangular bar with a halfcylinder profile contact surface, overcoming the manufacturing difficulties associated with a hemispherical punch. The radius of the punch head is $1.25 \mathrm{~mm}$ and the width of the punch is $8 \mathrm{~mm}$. The test specimen shown in Fig. 2 is a beam with rectangular cross section with width of $2 \mathrm{~mm}$ and height of $1.6 \mathrm{~mm}$. The height of the beam is slightly greater than the depth of the shallow slot, such that when the four fixing screws are tightened the beam sample is fully clamped. The central part of the beam is $8 \mathrm{~mm}$ and an additional $4 \mathrm{~mm}$ or more of material is included at both ends to hold the beam. Chamfers with size $0.2 \mathrm{~mm} * 45^{\circ}$ were created on the bottom slot to avoid stress singularity. The four screws were tightened with same torque using a torque wrench to keep the top and bottom surfaces parallel. The punch is guided by the slot in the top block. The bottom block is made of two pieces, making the chamber easier to manufacture. The top and bottom blocks and punch are made of stainless steel, while the tested specimen is aluminium AA2024 with chemical composition in weight percentage shown in Table 1 . The specimen material Young's modulus and Poisson's ratio are $73.1 \mathrm{GPa}$ and 0.3 , respectively. Lubricant was applied to the punch top surface and the side surface to reduce the friction between the punch and specimen and punch and the top block slot.

\section{Experiments}

The small punch beam testing tool system was fitted to a Denison Mayes material testing machine and the force applied to the

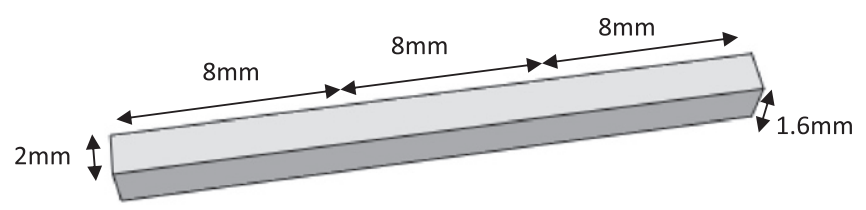

Fig. 2. Specimen for small punch beam test.

Table 1

Chemical composition of AA2024 in Wt.\%.

\begin{tabular}{llllll}
\hline Component & $\mathrm{Al}$ & $\mathrm{Mg}$ & $\mathrm{Si}$ & $\mathrm{Cr}$ & $\mathrm{Mn}$ \\
\hline Wt.\% & $90.7-94.7$ & $1.2-1.8$ & Max 0.5 & Max 0.1 & $0.3-0.9$ \\
Component & $\mathrm{Ti}$ & $\mathrm{Cu}$ & Zn & Fe & Other \\
Wt.\% & Max 0.15 & $3.8-4.9$ & Max 0.25 & Max 0.5 & Max 0.15 \\
\hline
\end{tabular}

punch and the displacement of the machine movement were recorded. The maximum speed of the press head was set to $1 \mathrm{~mm}$ per minute; therefore, the strain rate of the specimen is small and dynamic effects are not considered. The test was terminated when the press head displacement reached $0.9 \mathrm{~mm}$ and then the press head was released.

The recorded force and displacement were the force applied onto the specimen and displacement of the central point on the beam top surface: friction, punch weight and the elastic deformation of punch are assumed to be negligible. The typical punch force-displacement is shown in Fig. 3. The specimen initially deformed elastically in region A, followed by elastic and plastic deformation in region $B$. In region $C$, when large deformation occurs, damage is accumulated within the specimen and the material shows softening behavior with the total force decreasing. In the final region $\mathrm{D}$, specimen fails as the critical crack length is reached.

\section{FEM modelling}

\subsection{Geometry}

The aim of the investigation is to characterize material plastic properties through the comparison between numerical and experimental results. The Finite Element Method model shown in Fig. 4 consists of a specimen, a top block, a bottom block and a punch.

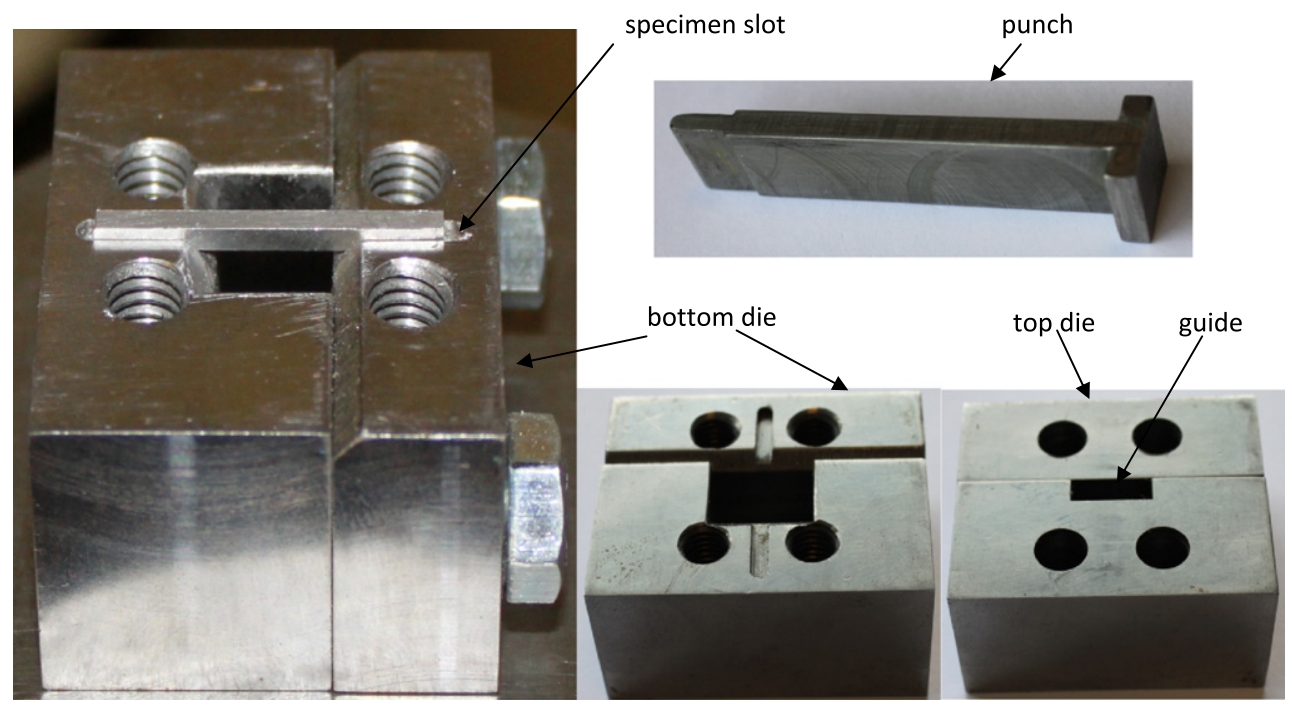

Fig. 1. Small punch beam test tool system. 


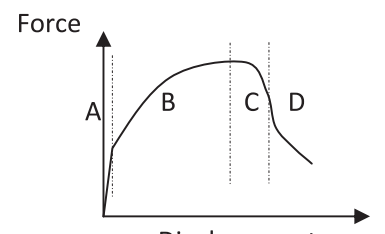

Displacement

Fig. 3. Typical small punch testing force vs. displacement curve.

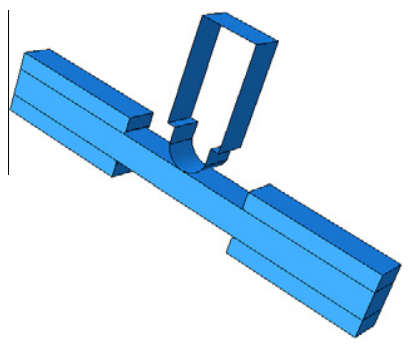

Fig. 4. FEM geometrical model.

The ABAQUS/implicit finite element code was used for the simulation. The steel punch is treated as a rigid body as it is significantly stiffer than the aluminium test specimen. The top and bottom blocks were modelled as elastic bodies. The length between the two inner surfaces of the bottom block is $8 \mathrm{~mm}$ and the width and the height of the beam cross section are $1.6 \mathrm{~mm}$ and $2 \mathrm{~mm}$, respectively.

\subsection{Material model}

As the specimen is aluminium AA2024-T3, an oxide layer was formed on its surface when it was exposed to the atmosphere. As this oxide layer is very thin, its influence on the mechanical properties of specimen was not considered. The multi-linear strain hardening material model shown in Fig. 5 was used in the ABAQUS numerical simulation, with S1, S2 and S3 representing the initial yield stress, proof stress (at $0.2 \%$ plastic strain) and ultimate tensile strength, respectively. The material will yield and plastic deformation occurs when von Mises stress reaches the yield surface, which is assumed to be a function of equivalent plastic strain. The von Mises stress $\sigma$ is represented as

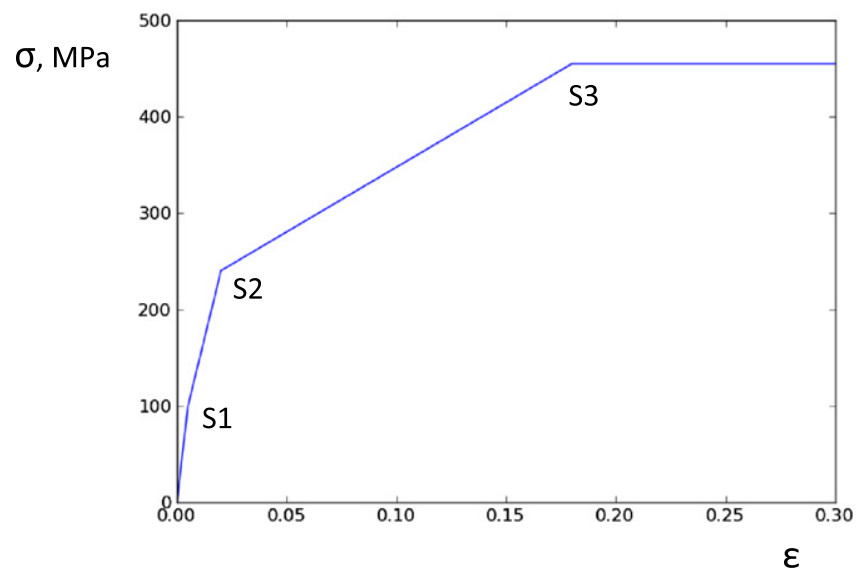

Fig. 5. Material stress vs. plastic strain relationship. $\sigma=\sqrt{\frac{1}{2}\left[\left(\sigma_{1}-\sigma_{2}\right)^{2}+\left(\sigma_{2}-\sigma_{3}\right)^{2}+\left(\sigma_{3}-\sigma_{1}\right)^{2}\right]}$

where $\sigma_{1}, \sigma_{2}$ and $\sigma_{3}$ are the principle stresses.

\subsection{Boundary conditions and loading}

Both top block and bottom blocks were fully constrained. The punch is assumed to be a rigid body and a reference point assigned to it in ABAQUS, such that only this reference point is required to be constrained. The three rotation degrees of freedom and the two translation degrees of freedom within the horizontal plane were set to 0 . The load applied to the specimen was replaced by vertical displacement control of the punch. The reaction force from the ABAQUS model corresponding to this constraint corresponds to the force applied to the specimen.

\subsection{Contact and friction coefficient}

Contact between the punch head and specimen top surface, specimen and top block and specimen and bottom block were considered in the ABAQUS model. The influence of the friction coefficient on the punch force versus the top surface middle point displacement was investigated. As lubricant was applied to punch top, the friction coefficient was assumed to be zero, while the friction coefficient between the top block and specimen, bottom block and specimen were finally set at 0.3 . The default penalty formulation in ABAQUS/Standard implicit codes was applied.

\subsection{Mesh convergence}

The ABAQUS three dimensional brick element C3D20R with reduced integration was used in the analysis. Four discretisation cases, as shown in Fig. 6(a) to (d), were investigated. The mesh used in characterization of material properties by the Genetic Algorithm method was selected by considering the CPU requirements and the accuracy of the analyses, as reported in Section 6.2.

\section{Material properties characterization using genetic algorithm method}

The Genetic Algorithm (GA) [20] optimization method is based on a simple natural rule: survival of the fittest. The fitter creatures will have more chances to survive and to produce off-spring. The GA method is applied to characterize the material nonlinear plastic parameters. The aim is to use the GA to search for the best values for a set of material parameters by comparing the finite element and experimental results. The total displacement is discretized uniformly into " $n$ " segments; therefore, without considering the initial point, there are " $n$ " values of displacement $u_{i}(i=1,2, \ldots, n)$ and their corresponding force values are $F_{i}(i=1,2, \ldots, n)$. However, as the experimental and finite element method results may not fall exactly at the discretized displacement point, a linear interpolation method is used to obtain the corresponding force value. Shown in Fig. 7, assuming $u_{i}$ falls within the experimental or finite element method consecutive points $u_{j}$ and $u_{j+1}$, the force $F_{i}$ corresponding to $u_{i}$ can be calculated as

$F_{i}=F_{j}+\frac{u_{i}-u_{j}}{u_{j+1}-u_{j}} *\left(F_{j+1}-F_{j}\right)$

A flow chart of a general GA optimization procedure is given in Fig. 8. Eq. (3) shows the formulation of the objective function $\Phi$, which is defined as the square root of the average of the summation of the square of the relative difference between the experimental and numerical results. The fitness is simply chosen as the inverse of the objective function, i.e. $f=\frac{1}{\Phi}$ 


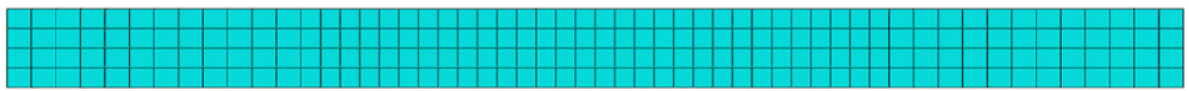

(a)

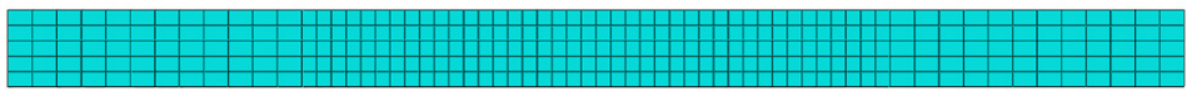

(b)

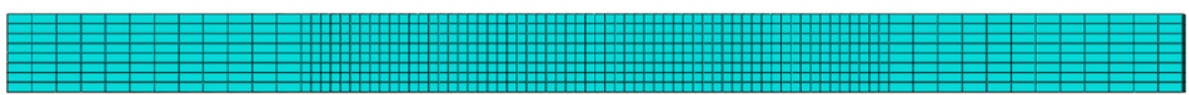

(c)

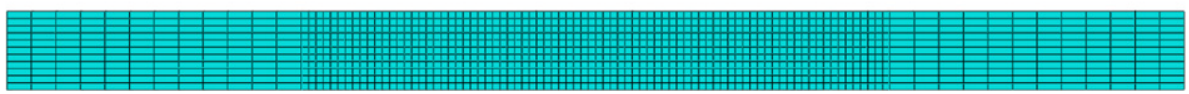

(d)

Fig. 6. FEM model with different mesh size near the beam center: (a) $0.4 \mathrm{~mm}$, (b) $0.3 \mathrm{~mm}$, (c) $0.2 \mathrm{~mm}$ and (d) $0.15 \mathrm{~mm}$.

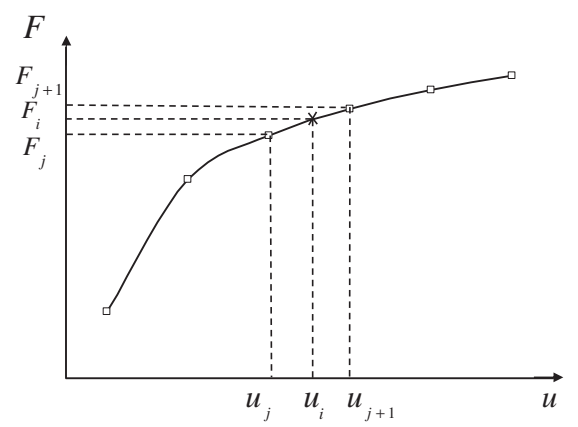

Fig. 7. displacements and their corresponding forces.

$\Phi=\sqrt{\frac{1}{n} \sum_{i=1}^{n}\left(\frac{F_{i}^{c a l}-F_{i}^{\exp }}{F_{i}^{\exp }}\right)^{2}}$

where $F_{i}^{\text {exp }}$ and $F_{i}^{c a l}$ are the experimental and finite element method punch forces corresponding to the $i$ th displacement $u_{i}$. For this case, the linear interpolation method was used.
Smaller values of the objective function correspond to the larger values of the fitness. The general GA procedure consists of initialization, selection, crossover and mutation, with a higher fitness seed having more chance to be selected and one of the fittest offspring at each generation always being kept. The GA procedure stops when the convergence criterion is satisfied.

One example of selection, cross over and mutation from generation 0 to generation 1 of the GA method, is described as follows. As the material properties to be characterized are S1, S2 and S3, the range of their values are set at start. For initialization, S1, S2 and S3 are randomly assigned an integer from 0 to 255 . At the start, chromosomes with the integers from 0 to 255 are represented as 8 digit binary strings. At iteration 0 , there are 20 seeds for each set of S1, S2 and S3, and each seed can be linearly mapped to the parameters S1, S2 and S3 used in the FE models, as shown in Fig. 9. These 20 seeds are ranked according to the objective function value calculated from the corresponding FE analysis: the seed with the smallest objective function ranks first, while the seed with the largest objective function ranks last. The selection operator is to delete the last seed for preparation of the next procedure cross over. The cross over procedure randomly selects a pair of seeds from seed numbers 1 to 18 and exchanges their genes at a random position. This procedure is continued until all seeds from seed

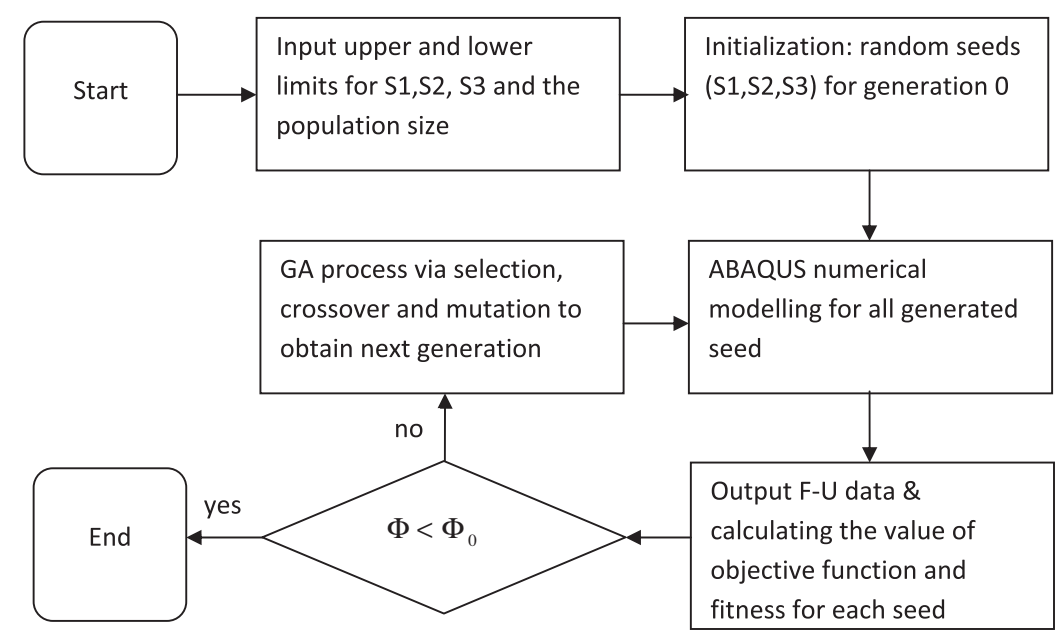

Fig. 8. Flow chart of the genetic algorithm. 


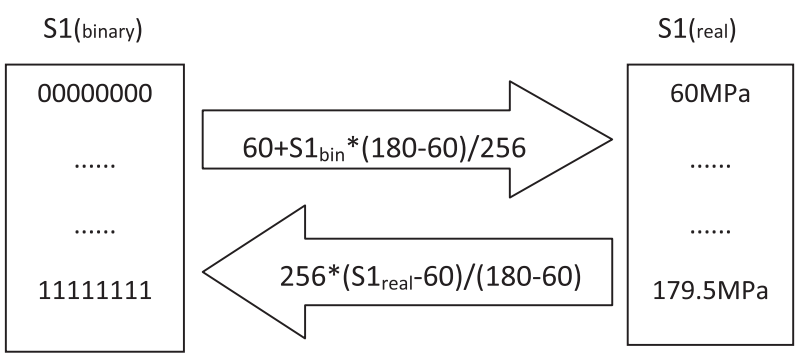

Fig. 9. Mapping parameters from integers to real.

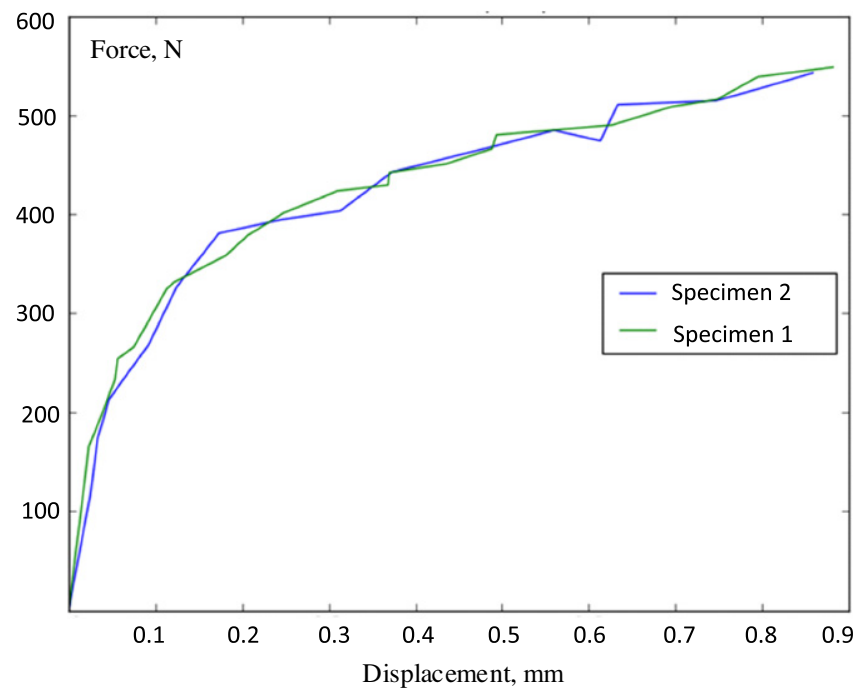

Fig. 10. The two experiment force vs. displacement results.

Table 2

Comparison of punch force at different mesh size.

\begin{tabular}{lrccl}
\hline Case & 1 & 2 & 3 & 4 \\
\hline Mesh size (mm) & 0.4 & 0.3 & 0.2 & 0.15 \\
Force applied onto specimen (N) & 455.12 & 495.428 & 500.601 & 500.398 \\
Relative difference to the finest & 9.05 & 0.99 & -0.04 & NA \\
$\quad$ mesh (case 4)\% & & & & \\
\hline
\end{tabular}

Table 3

Influence of friction coefficient on the punch force at top surface middle point displacement of $0.85 \mathrm{~mm}$.

\begin{tabular}{lrrrr}
\hline Friction coefficient & 0.1 & 0.2 & 0.3 & 0.4 \\
Punch force, N & 490.9 & 500.0 & 508.7 & 513.9 \\
\hline
\end{tabular}

Table 4

Objective function $\Phi$ varies with iteration number.

\begin{tabular}{llllll}
\hline Iteration No & 0 & 5 & 10 & 15 & 20 \\
Objective function $\Phi$ & 0.0817 & 0.0749 & 0.0731 & 0.0686 & 0.0612 \\
\hline
\end{tabular}

number 1 to 18 are selected and crossed-over, giving new seeds numbered from 1 to 18 . Finally, three seeds from 0 to 18 are randomly selected and mutated at random positions to obtain S1, S2

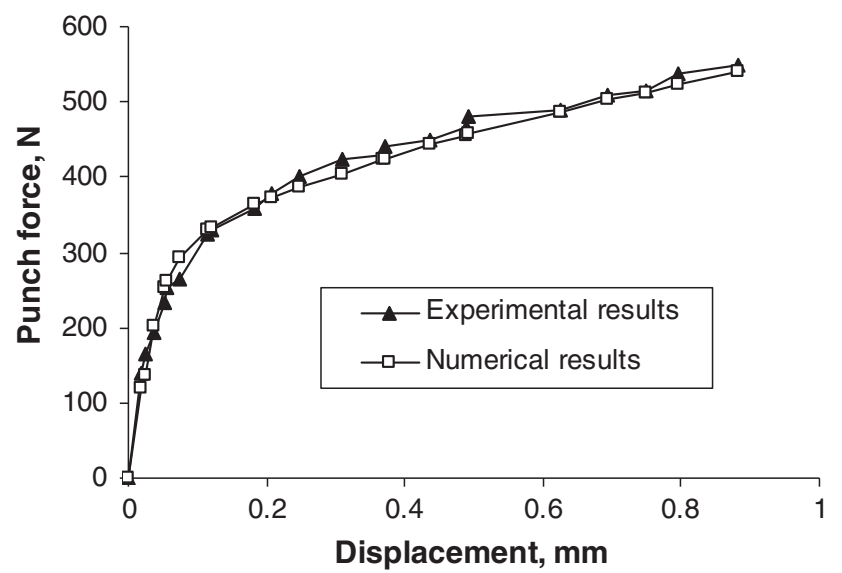

Fig. 11. Comparison between experiment and FEM numerical force vs. displacement results.

and S3 respectively and the newly generated seed is numbered as 19. 20 new seeds for the next iteration are then prepared.

\section{Results}

\subsection{Experimental results}

Two tests were performed and the test results are shown in Fig. 10. Due to the accuracy of the testing machine, the testing results fluctuate. However, from this figure, it can be seen clearly that the test results are quite consistent. In this paper, we aim to present a novel design and a GA method to characterize material plastic parameters; therefore, only the specimen 2 results were used for this purpose. To make the comparison easier, the average experimental results were compared with FEM results.

\subsection{Finite element results}

Fig. 6(a) to (d) show the meshes with element size of $0.4 \mathrm{~mm}$, $0.3 \mathrm{~mm}, 0.2 \mathrm{~mm}$ and $0.15 \mathrm{~mm}$ for the central part of the beam. Table 2 displays the punch force corresponding to punch vertical displacement of $0.9 \mathrm{~mm}$ under different element size. The relative difference of the reaction force to the finest mesh is also shown in this table. From this table, it can be concluded that the model with mesh size of $0.2 \mathrm{~mm}$, Fig. 6(c), gives a good balance between accuracy and CPU time and the results reported here were based on this mesh.

To investigate the influence of the friction coefficient on the punch force-displacement curve, different friction coefficients between upper die and specimen and lower die and specimen were assigned to the FE model. The simulation results are shown in Table 3, from which it can be seen that the punch force increases with increasing of friction coefficient. By comparing these results, it is found that the maximum relative difference is less than $4.5 \%$. In this paper, friction coefficient of 0.3 is used.

\subsection{GA method results}

Table 4 shows the history of evolution of the minimum objective function $\Phi$ of generation varying with iteration number. At the beginning of the GA procedure, all seeds are randomly generated and the lowest objective function value is 0.0817 . The binary string for the best seed is [01011101], [01000100] and [00000011] and their corresponding S1, S2 and S3 values are $103.6 \mathrm{MPa}$, 239.8 MPa and 401.4 MPa. From Table 4, it can be seen that gener- 


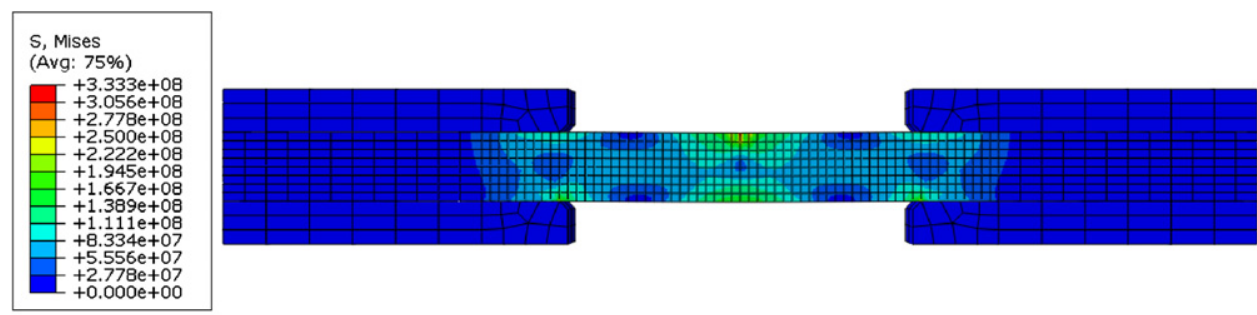

(a)
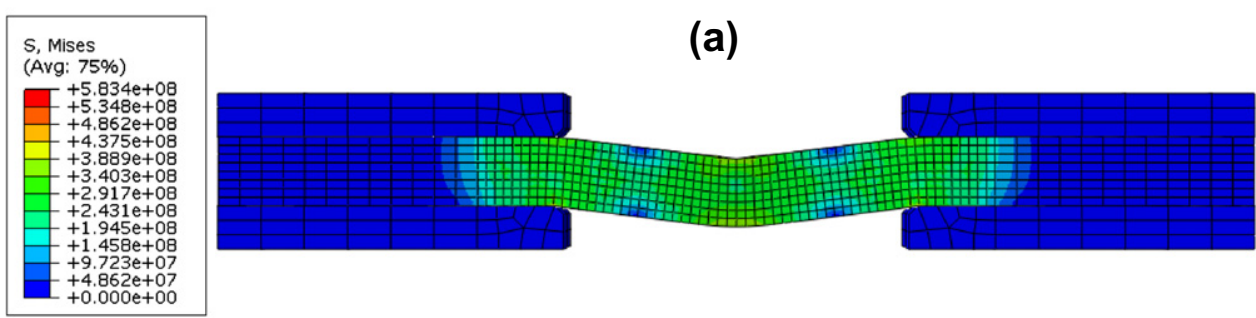

(b)
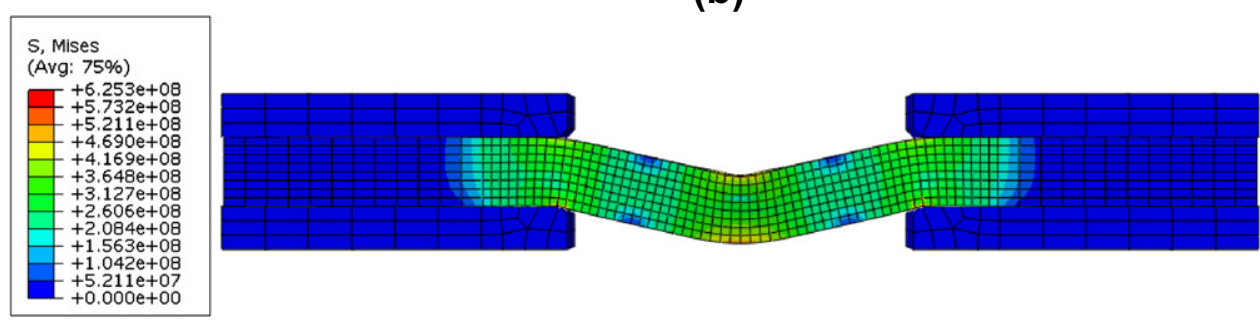

(c)

Fig. 12. The von Mises stress contours of the specimen at different top surface middle point displacement: (a) $0.025 \mathrm{~mm}$, (b) $0.5 \mathrm{~mm}$ and (c) $0.89 \mathrm{~mm}$.

ally the minimum value of the objective function decreases as the iteration number increases. The value of the minimum objective function will eventually reach a certain value, leading to the convergence of the GA. In this paper, after evolution of 20 generations, the minimum $\Phi$ reaches 0.0612 . The binary strings for this generation are [01011010], [00100010] and [11101010] and their corresponding S1, S2 and S3 values are $102.2 \mathrm{MPa}, 219.9 \mathrm{MPa}$ and $509 \mathrm{MPa}$ respectively. The ultimate tensile strength is very close to the $496 \mathrm{MPa}$ report in Ref. [21].

Comparison of the experimental and finite element punch force-displacement curves is shown in Fig. 11, from which it can be seen that the predicted results are in good agreement the experimental results.

After obtaining the material plastic parameters, the FEM simulation results produced using the optimal parameters values were re-investigated. Fig. 12(a) to (c) show the stress contours at the top surface middle point displacement of $0.025 \mathrm{~mm}$, $0.5 \mathrm{~mm}$ and $0.89 \mathrm{~mm}$, respectively. From Fig. 12(a), it can be seen that apart from the contact point between punch and specimen, the maximum stress was less than the $0.2 \%$ proof stress and the high stresses occurred in elements close to the middle points of the top and bottom surfaces. This shows the beam deformation behaviour. By checking the punch force versus specimen top surface middle point displacement, it is found that this stage corresponds to linear elastic deformation in the specimen. With higher punch force applied onto the specimen, the maximum von Mises stress exceeded the yield stress and plastic deformation developed in the specimen. From Fig. 12(b) and (c), it is clear that the location of high stress in the elements expanded from the middle points of the top and bottom surfaces to the centre of the specimen and the areas near the bottom support regions also have high stresses.

\section{Conclusions and future work}

Based on the above results, it is concluded that:

(1) The novel small punch beam testing tool system presented has an advantage over conventional tensile testing in that much less sample material is required. It also has an advantage over the semispherical head small punch test, as the cylindrical shape punch head is much easier to manufacture with high accuracy and low cost.

(2) As the test specimen are smaller than the conventional SPT specimen, the same amount of material removed from an in-service component could make more specimens, or, to produce same number of specimens will require less material to be removed from components.

(3) The small punch beam testing results are consistent, making the testing method a suitable candidate method for material property characterization.

(4) The GA method has been successfully used to characterize the material plastic parameters. The calculated punch force-displacement results are in good agreement with experimental results.

For future work, beam specimens will be tested to failure and characterization of material damage parameters will be investigated. If this can be achieved it would enable numerical estimation of material fracture toughness. The beam dimensions could also be further reduced to thickness of $0.5 \mathrm{~mm}$, width of around $1 \mathrm{~mm}$ and length of 6 to $8 \mathrm{~mm}$. This will require less material compared to the beam specimens used in this research. Other methods, such as the neural network method, will also be used to characterize material property parameters. 


\section{Acknowledgements}

The author Xingguo Zhou would like to thank the financial support by Scottish Overseas Research Student Awards Scheme and University of Strathclyde.

\section{References}

[1] Manahan MP, Argon AS, Harling OK. The development of mininaturized disk bend test for the determination of postirradiation mechanical properties. Journal of Nuclear Materials 1981;103\&104:1545-50.

[2] Egan Patrick, Whelan Maurice P, Lakestani Fereydoun, Connelly Michael J. Small punch test: an approach to csolve the inverse problem by deformation shaper and finite element optimization. Comput Mater Sci 2007;40:33-9.

[3] Isselin J, Iost A, Golek J, Najjar D, Bigerelle M. Assessment of the conbstitutive law by inverse methodology: small punch test and hardness. J Nucl Mater 2006;352:97-106.

[4] Steven M, Foulds Jude R, Jewett Charles W, Srivastav Sanjeev, Edidin Avram A Validation of a small punch testing technique to characterize the mechanical behaviour of ultra-high-molecular-weight polyethylene. Biomaterials 1997; 18:1659-63.

[5] Karthik V, Visweswaran P, Vijayraghavan A, Kasiviswanathan KV, Raj Baldev. Tensile-shear correlations obtained from shear punch test technique using a modified experimental approach. J Nucl Mater 2009;393(3):425-32.

[6] Milicka Karel, Dobes Ferdonand. Small punch testing of P91 steel. Int J Pressure Vessels Piping 2006:83:625-34.

[7] Kobayashi Ken-ichi, Tabuchi Masaaki, Stratford Gavin C. Creep Rupture life of welding components in P92 Ferritic steel using small punch creep test. Metal J 2010; LXIII:54-8.

[8] Blagoeva DT, Hurst RC. Application of the CEN (European Committee for Standardization) small punch creep testing code of practice to a representative repair welded P91 pipe. Mater Sci Eng 2009;A 510-511:219-223.
[9] Mao X, Takahashi H. Development of a further-miniaturized specimen of $3 \mathrm{~mm}$ diameter for tem disk small punch tests. J. Nucl Mater 1987;150:44-52.

[10] Foulds J, Viswanathan R. Small punch testing for determining the material toughness of low alloy steel components in service. J Eng Mater Technol 1994;116(4):457.

[11] Li Yingzhi, Hurst Roger, Matocha Karel, Cizek Peter, Blagoeva Darina. New approach to determine fracture toughness from the small punch test. Metal J 2010;LXIII:94-102.

[12] Takahashi H, Shoji T, Mao X, Hamaguchi Y, Misawa T, Saito M Oku T, Kodaira T, Fukaya, K Nishi H, Suzuki M. Recommended practice for small punch (SP) testing of metallic Materials, JAERI-M; September 1988: 88-172.

[13] CEN, Small punch test method for metallic materials. Part A: A Code of Practice for Small Punch Creep, 2005.

[14] CEN, Small punch test method for metallic materials. Part B: A Code of Practice for Small Punch Testing for Tensile and Fracture Behavior, 2006.

[15] ASTM F2183-02(2008) Standard test method for small punch testing of ultrahigh molecular weight polyethylene used in surgical implants.

[16] Guan Kai-shu, Wang Zhi-wen, Xu Tong, Shou Bi-nan. Development of small punch test in China. Czech Metal J 2010;LXIII.

[17] Pan W, Boyle Jim, Ramlan Mohd, Dun Craig, Ismail Mohd, Hakoda Kenji. Selected for Czech Metallurgical Journal 2010; LXIII:174-9.

[18] Sehgal DK, Husain A, Pandey RK. The Rectangular Shaped Miniature Specimen to study the Mechanical Behavior of Materials. In: 2nd international conference on Mechanical and electronics engineering (ICMEE), vol. 1, Kyoto, 2010; 196-9.

[19] Abendroth M, Kuna M. Determination of deformation and failure properties of ductile materials by means of the small punch test and neural networks. Comput Mater Sci 2003;28:633-44.

[20] Castro CF, Antonio CAC, Sousa LC. Optimisation of shape and process parameters in metal forging using genetic algorithms. J Mater Process Technol 2004;146:356-64.

[21] http://wenku.baidu.com/view/b70b8a5c3b3567ec102d8a44.html [accessed 03.10.10]. 